\title{
Performance Evaluation of a Loeb-Eiber Mass Filter at 1 Torr
}

\author{
William D. Hoffmann, ${ }^{1}$ Feng Jin, ${ }^{2}$ Randall E. Pedder, ${ }^{3}$ Christopher Taormina, ${ }^{3}$ \\ Glen P. Jackson ${ }^{1,2}$ \\ ${ }^{1}$ Department of Forensic and Investigative Science, West Virginia University, Morgantown, WV 26506, USA \\ ${ }^{2}$ C. Eugene Bennett Department of Chemistry, West Virginia University, Morgantown, WV 26506, USA \\ ${ }^{3}$ Ardara Technologies LP, Ardara, PA 15615, USA
}

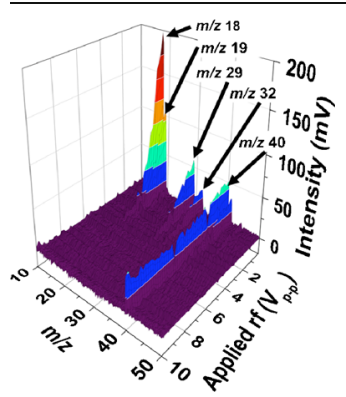

Abstract. The Loeb-Eiber mass filter is best operated at relatively high pressures-such as 1 Torr-where collisional dampening of ions up to the mass filter thermalizes the ions' kinetic energy, which is a requirement for effective filtering. The inter-electrode gaps of $\sim 8 \mu \mathrm{m}$ require if amplitudes on the order of $0-5 \mathrm{~V}_{p-p}$ at approximately $50 \mathrm{MHz}$ to achieve mass filtering up to $\mathrm{m} / \mathrm{z} 40$. Mass filtering between the $25-\mu \mathrm{m}$ diameter wires, therefore, takes place on time frames less than the collision frequency at $\sim 1$ Torr. The low power and high pressure capabilities of the Loeb-Eiber mass filter make it ideally suited for miniaturization, where power and space are a premium. In the present work, a Loeb-Eiber mass filter was constructed using commercial silicon-on-insulator (SOI) microfabrication techniques. Ions transmitting through the chip-based Loeb-Eiber mass filter were characterized in real time using a traditional linear quadrupole mass analyzer in series with the Loeb-Eiber mass filter. The new hybrid instrument has enabled us to verify several important claims regarding the operation of the Loeb-Eiber mass filter: (1) that ions can be effectively filtered at $\sim 1$ Torr, (2) that for ions of a fixed mass-to-charge ratio, the ion transmission current decreases linearly with increasing if amplitude on the Loeb-Eiber mass filter, (3) that the cutoff voltage at which all ions of a particular $\mathrm{m} / \mathrm{z}$ value are effectively blocked is linearly related to mass-to-charge, and (4) that square waveforms can filter ions more effectively than sinusoidal waveforms for a given peak-to-peak if amplitude.

Keywords: Portable, Microfabricated, Miniature

Received: 26 September 2014/Revised: 30 October 2014/Accepted: 31 October 2014/Published Online: 20 December 2014

\section{Introduction}

$\mathrm{T}$ here are two major obstacles to miniaturizing mass spectrometers: (1) the requirement for high vacuum (e.g., $<10^{-4}$ Torr), which requires bulky, power-drawing vacuum pumps, and (2) the use of high voltages (e.g., >1000 $\mathrm{V}_{\mathrm{ac}}$ ), which requires sizable high-voltage power generators/amplifiers and matching circuits [1, 2]. If mass spectrometers could be made to operate at higher pressures and with reduced power consumption, their physical footprint could be noticeably reduced. Because of the possibility to mass-produce miniature instruments at a fraction of the cost of typical bench-top mass spectrometers, the potential exists to significantly impact homeland security, national defense, forensic investigations, and high school- or university-level education programs.

Correspondence to: Glen P. Jackson; e-mail: glen.jackson@mail.wvu.edu
Related to the rapidly growing interest in field- and person-portable mass spectrometers [3], the forensic science community has also shown an active interest in pursuing a variety of applications using portable mass spectrometers [4-7]. Terrorism and security concerns have precipitated an increased awareness for the ability of mass spectrometers to be broad-spectrum threat detection devices. For example, upon making recommendations to the Transportation Security Administration (TSA), the National Research Council (NRC) recently announced that "the TSA should establish mass spectrometry as a core technology for identifying a broad array of explosives as well as chemical and biological agents" [8]. This trend has been supported through a National Science Foundation (NSF)-sponsored workshop in 2003 on "Prospects for Miniaturization of Mass Spectrometry" and more recently through a 2012 workshop on "Strengthening Forensic Science through connections with the Analytical Sciences." The workshop report, titled "Science on Location: Forensic Science on the Move," 
encourages the development of portable mass spectrometry for forensic applications [9].

Cotter and coworkers have developed a miniature TOF with an adjustable reflectron electrode $[10,11]$. The earliest manifestation of this instrument achieved a resolving power of 210 at $\mathrm{m} / \mathrm{z} 1061$ [10], whereas later improvements increased the resolving power to 330 [11]. Wapelhorst et al. developed a miniature TOF with a $5 \mathrm{~mm} \times 10 \mathrm{~mm}$ footprint that could operate at $\sim 8$ mTorr [12], and Verbeck's group has demonstrated the use of microelectromechanical systems (MEMS) technology to develop considerably smaller electrodes for use in a variety of mass analyzers [13].

Taylor's group at the University of Liverpool has made significant improvements in the area of miniaturized linear quadrupole instruments [14-17]. Initial improvements used gold-plated borosilicate glass electrodes precisely aligned with silicon substrates to investigate He/air mixtures [14]. Later work by others employed MEMS techniques to produce quadrupoles on the order of $0.5-1 \mathrm{~mm}$ diameter rods with resolving powers between of 20 and 200 in the low mass range $(<\mathrm{m} / \mathrm{z}$ 219) $[15,18]$. Using a portable mass spectrometer based on a quadrupole mass filter (QMF), Taylor's group were able to monitor the respiratory gases and volatile anesthetic agents from anesthetized equine patients and thereby provide limits of detection that were up to two orders of magnitude better than traditional IR-based monitoring for real-time breath analysis [16].

Ion trap mass analyzers have also been the subject of miniaturization [3]. In contrast to TOFs, linear quadrupoles, and magnetic sectors [19], ion traps enjoy the ability to perform tandem mass spectrometry with a single mass analyzer [20]. The simplified geometry of a cylindrical ion trap in comparison to $3 \mathrm{D}$ ion traps has led to a significant research investment in the miniaturization of ion traps [21-26] and micro-fabricated ion trap arrays [27-29]. Lammert [30, 31] and Austin [32-34] have studied toroidal ion traps of various geometries in an attempt to expand the quantitative dynamic range of ion traps in miniature and portable instruments.

The operating principles of the Loeb-Eiber filter used in this study has been described elsewhere [35-38]. In short, the filter is comprised of an array of interleaved or interdigitated wires that are each 20-100 $\mu \mathrm{m}$ in diameter. Although the Loeb-Eiber filter is similar in construction to a Bradbury-Nielsen Gate (BNG) [39-42], the Loeb-Eiber filter has wires that are closer together than the diameter of each wire and employs a very high frequency $(40-100 \mathrm{MHz})$ waveform instead of low frequency pulsed DC potentials. Scanning the rf amplitude on the Loeb-Eiber array of wires scans the low mass cutoff (LMCO), which ultimately enables a spectrum to be collected.

Because mass filtering occurs in a single pass between the $\sim 25 \mu \mathrm{m}$ wires, the Loeb-Eiber mass filter has at least two major benefits over existing mass spectrometers, and several disadvantages. Regarding advantages, the Loeb-Eiber filter can operate at pressures in the region of 1 Torr $(\sim 1 \mathrm{mbar})$ and with operational waveforms on the order of $25 \mathrm{~V}_{p-p}$. One stage of pumping and simple electronic circuit boards should suffice to operate such a chip-based filter. The operating conditions of the Loeb-Eiber filter, therefore, enable considerably smaller mechanical and electronic components and less power consumption, all of which enable a smaller, lighter payload than existing mass filters. The major disadvantage of the Loeb-Eiber mass filter is that it currently only operates in a low-mass-cutoff mode, so mass selective filtering has not been demonstrated at this time. Ion masses therefore have to be determined by scanning the low mass cutoff, much as one would operate an rfonly quadrupole to collect a mass spectrum. The experiments herein describe the coupling of traditional linear quadrupole mass analyzer downstream of a Loeb-Eiber mass filter to enable mass-selective diagnosis of the ion beam transmitting through the Loeb-Eiber filter under different conditions.

\section{Experimental}

The Loeb-Eiber mass filter used in this work was produced using the multi-user MEMS process (MUMPS) (MEMSCAP Inc., Durham, NC, USA) [43-46]. The MEMS based mass filter had square cross-section electrodes of $25 \mu \mathrm{m} \times 25 \mu \mathrm{m}$ with an $8 \mu \mathrm{m}$ interelectrode spacing. The two interdigitated electrodes are electrically isolated from one another, rather like the electrodes of a capacitor, allowing rf voltages to be applied to either half of the mass filter. In the original concept of the device, sinusoidal rf voltages of equal magnitude with $180^{\circ}$ phase shift were applied to either half of the mass filter.

The performance of the Loeb-Eiber mass filter was evaluated by placing the mass filter in front of a traditional linear quadrupole mass analyzer, as shown in the $3 \mathrm{D}$ schematic in Figure 1. A custom fabricated glow discharge composed of a copper feedthrough was used as the ionization source and was operated at 0.6-1 Torr of Ar (Matheson TRIGAS, Fairmont, WV, USA), depending on the experiment. The customfabricated anode, steering electrode, and skimmer were made of stainless steel. The glow discharge and Loeb-Eiber mass filter are at a common pressure ( 1 Torr) and the mass filter

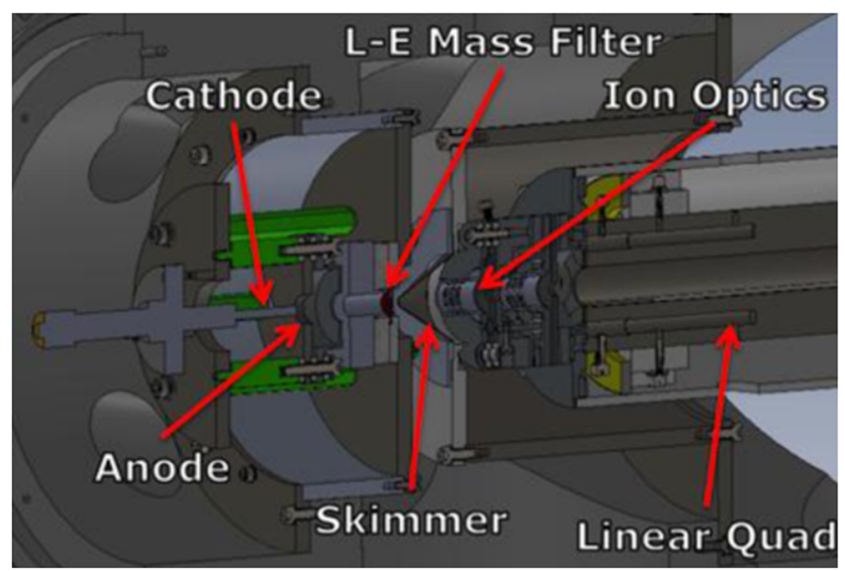

Figure 1. 3D schematic of the hybrid Loeb-Eiber/linear quadrupole instrument 
serves as the conductance limit to a differentially pumped intermediate pressure region ( $\sim 100 \mathrm{mTorr})$ using the drag stage of a SplitFlow turbo pump (Pfeiffer Vacuum, Nashua, NH, USA). Behind a 2-mm SS skimmer cone is a turbo-pumped high vacuum region $\left(\sim 10^{-5}\right.$ Torr) containing the commercially available ion optics, a 12-mm linear quadrupole, detection system, and Tempus software (all from Ardara Technologies, Inc., Ardara, PA, USA). Although this hybrid system includes many components that are not essential to the operation of a future portable Loeb-Eiber-based instrument, the hybrid system enables us to diagnose the transmitted ion signal through the Loeb-Eiber mass filter, which still operates at the relatively high pressure of $\sim 1$ Torr.

From an electrical engineering perspective, the microfabricated chip acts like a $\sim 200 \mathrm{pF}$ capacitor (depending on the specific connections). To apply very high frequency (VHF) rf waveforms to such a device is therefore not trivial. To avoid reflected losses, the rf voltage was taken from the arbitrary function generator (3252 AFG; Tektronix, Beaverton, OR, USA), amplified by a broadband $50 \mathrm{~dB}$ rf amplifier (525LA; E\&I, Rochester, NY, USA), then sent to a surface mount transformer (Mini-Circuits ADT9-1T; Brooklyn, NY) where the splitting and phase shifting took place. This bipolar signal, shifted by $180^{\circ}$, was applied to each half of the mass filter via gold contact pads in a custom-made mounting device [38]. The input amplitude of the $40 \mathrm{MHz}$ sinusoidal rf signal was then scanned linearly from $0-150 \mathrm{mV}_{p-p}$. The output of the ADT9-1T chip was measured offline to be $0 \sim 5 \quad \mathrm{~V}_{p-p}$ under similar conditions, which showed little to no reflected losses in signal transmission. Reflected losses increased with frequency above $45 \mathrm{MHz}$.

Experiments were conducted in two ways. First, a fixed rf frequency and amplitude was applied to the Loeb-Eiber mass filter, resulting in a fixed low mass cutoff (LMCO). During the fixed amplitude and frequency operation of the Loeb-Eiber filter, the linear quadrupole was scanned repeatedly across the range $m / z 10-50$ to acquire an averaged mass spectrum. The Loeb-Eiber filter could then be set to different rf amplitudes for subsequent characterization. In a second mode of operation, the rf amplitude applied to the Loeb-Eiber mass filter was repeatedly scanned at $5 \mathrm{~Hz}$ in a linear amplitude fashion while the quadrupole was operated in selected (single) ion monitoring (SIM) mode. The quadrupole was set to monitor different ions (e.g., $m / z$ 18, 29, 40, and 41) under different scanning parameters of the Loeb-Eiber mass filter. Spectra were typically averaged for $1 \mathrm{~min}$ each, unless otherwise noted.

\section{Results and Discussion}

Initially, the Loeb-Eiber mass filter was operated with a fixed frequency $(40 \mathrm{MHz})$ and selected amplitudes while the linear quadrupole was scanned from $\mathrm{m} / \mathrm{z} 10$ to 50 . The effect of $\mathrm{rf}$ amplitude on transmitted ion signal was determined at $\mathrm{rf}$ amplitudes from 0.1 to $10 \mathrm{~V}_{p-p}$ in $0.1 \mathrm{~V}_{p-p}$ increments, in random order. The glow discharge was operated at 1 Torr Ar in current- limited mode at $0.550 \mathrm{~mA}$. Figure 2 shows the change in ion signal as a function of applied rf voltage (top panel), as measured by the quadrupole mass filter.

At $0 \mathrm{~V}_{p-p}$, the intensity of $\mathrm{m} / \mathrm{z} 18$ is relatively high and constant until $0.8 \mathrm{~V}_{p-p}$ on the Loeb-Eiber filter. Above 0.8 $\mathrm{V}_{p-p}$, the signal intensity for $\mathrm{m} / \mathrm{z} 18$ decreases somewhat linearly to $3.0 \mathrm{~V}_{p-p}$ applied rf voltage. This result is in good agreement with the expected linear decrease in signal intensity, from first principles [36-38]. As expected from first principles, the signal intensity decreases at a different rate and with different cutoff value for ions with different $m / z$ values. For example, observe the change in intensity ratio of $m / z 40 / 18$ in the bottom panels of Figure 2 for the mass spectra collected at 0.8 and $3.0 \mathrm{~V}_{p-p}$, respectively, on the Loeb-Eiber mass filter. At $0.8 \mathrm{~V}_{p-p}$ on the Loeb-Eiber mass filter, the intensity ratio of $\mathrm{m} / \mathrm{z} 40 / 18$ measured by the quadrupole is $\sim 0.32$. At $3.0 \mathrm{~V}_{p-p}$ on the Loeb-Eiber mass filter, the low mass water peak is more severely attenuated such that the ratio of the same transmitting ions is now $\sim 2.1$. At $4.0 \mathrm{~V}_{p-p}$ on the Loeb-Eiber mass filter, the water peak at $\mathrm{m} / \mathrm{z} 18$ is insignificant relative to the noise, so is effectively cut off, but the argon ion peak at $\mathrm{m} / \mathrm{z} 40$ is still observable at approximately $50 \%$ its original abundance.

The cutoff voltage is defined as the rf amplitude above which the ion oscillation would exceed half the interelectrode spacing, effectively filtering ions of that $\mathrm{m} / \mathrm{z}$ value. With our experimental setup, a small number of ions are formed between the Loeb-Eiber mass filter and the skimmer, as demonstrated by the presence of residual background signal at $m / z 18$, above the cutoff voltage. The result is a small, constant background current of $\mathrm{m} / \mathrm{z} 18$ that could not be totally attenuated at any applied rf voltage on the Loeb-Eiber filter because the ions are formed downstream of the Loeb-Eiber mass filter. Proper background correction could alleviate this

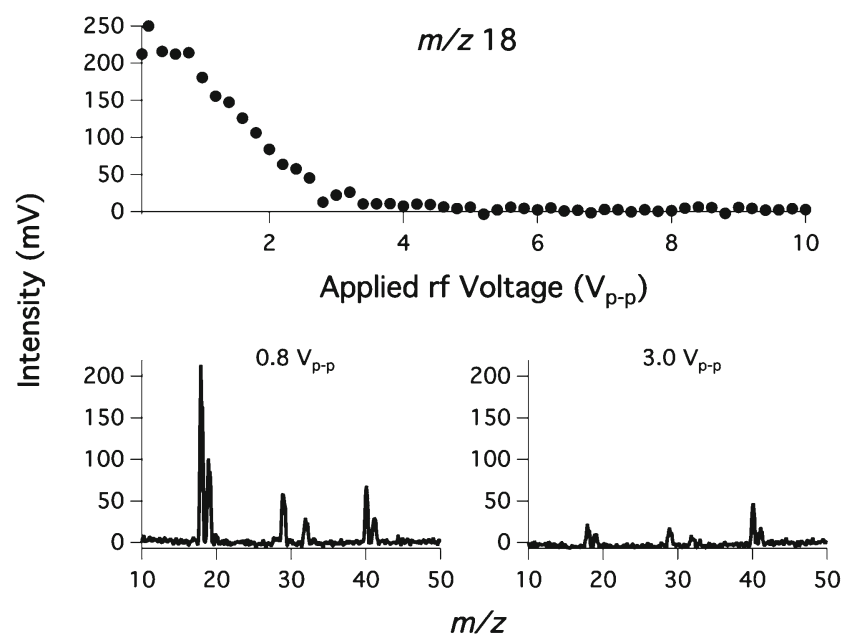

Figure 2. Intensity of $\mathrm{m} / \mathrm{z} 18$ measured by the quadrupole mass filter as a function of selected rf amplitudes on the Loeb-Eiber mass filter (top panel). The points were collected in random order. Bottom panels show the mass spectra obtained by the quadrupole at 0.8 and $3.0 \mathrm{~V}_{p-p}$ applied if at $40 \mathrm{MHz}$ on the Loeb-Eiber filter 
problem in the future. This steady-state mode of operation could be beneficial for limiting unwanted low-mass ions from a beam containing a distribution of sizes of ions.

The second mode of operation of the hybrid Loeb-Eiber/ linear quadrupole instrument is implemented by scanning the applied rf amplitude (and therefore, the LMCO) on the LoebEiber mass filter while operating the quadrupole in SIM mode.

Figure 3 shows the intensity of $m / z 18,29,40$, and 41 measured by the quadrupole as a function of the applied $\mathrm{rf}$ amplitude on the Loeb-Eiber mass filter. The data shown in Figure 3 have been boxcar-averaged from 10,000 to 100 points. For each selected ion, the transmission current shows a region of linear decline with increasing rf amplitude, as shown with fitted linear regression lines. These linear regions can be extrapolated to the $\mathrm{x}$-axis to provide idealized cutoff times or cutoff voltages (if calibrated), which are plotted in Figure 4. The excellent linearity of the extrapolated cutoff points with respect to $\mathrm{m} / \mathrm{z}$ demonstrates that fundamentally, the Loeb-Eiber mass filter is working according to theory.

Originally proposed by Eiber [36, 47], the equation governing the amplitude of ion motion is given by Equation (1):

$A=\frac{E_{q}}{m \omega^{2}}$

where $\mathrm{A}$ is the amplitude of ion motion, $\mathrm{E}$ is the electric field strength, $\mathrm{q}$ is the charge on the ion, $\mathrm{m}$ is the mass of the ion, and $\omega$ is the angular frequency of the applied rf voltage. With a fixed inter-electrode spacing, there is a linear relationship between the amplitude of applied rf voltage and the amplitude of ion oscillation. Theoretically, the maximum amplitude needed to filter an ion is half the inter-electrode spacing and when this voltage is applied, it is termed the cutoff voltage for a particular $\mathrm{m} / \mathrm{z}$ ion. From Equation (1), there is also a linear relationship between the applied voltage and the $m / z$ value that is effectively cut off. This is illustrated in Figure 4 by the linear relationship between cutoff time and $\mathrm{m} / \mathrm{z}$. Because the rf amplitude is increased linearly with

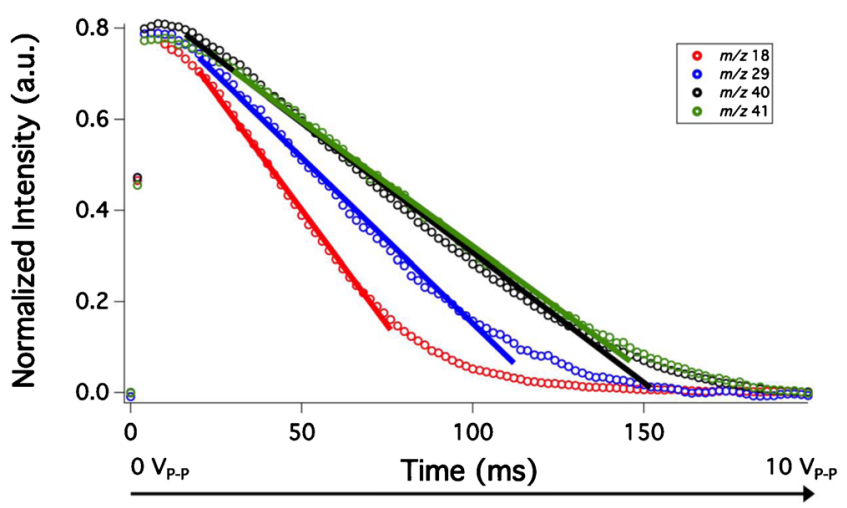

Figure 3. Boxcar averaged signal for $m / z$ 18, 29, 40, 41 acquired by the quadrupole in SIM mode while the amplitude (LMCO) of the Loeb-Eiber mass filter is scanned linearly at $5 \mathrm{~Hz}$. The linear regression lines were fitted over the range where the first derivative is less than 0.05

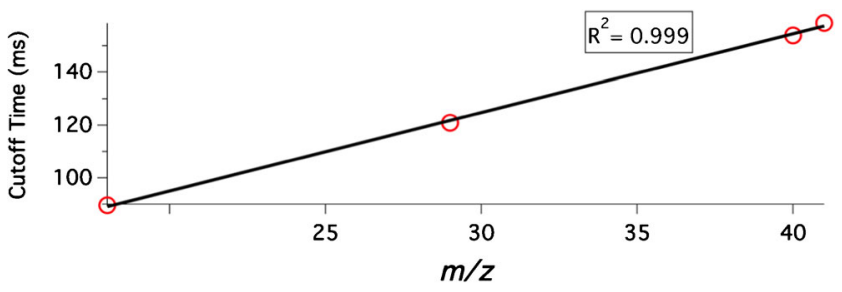

Figure 4. Demonstration of the linear relationship between $\mathrm{m} / \mathrm{z}$ and cutoff time. Because the if amplitude is increased linearly as a function of time, there is also a linear dependence between $\mathrm{m} / \mathrm{z}$ and cutoff amplitude

time, there is a linear relationship between the applied rf amplitude and the $m / z$ value that is cut off.

In reality, the ion profiles undergo curvature near their respective cutoff points, and this makes it considerably more difficult to establish the exact cutoff points in the absence of a secondary mass-selective detector. To deconvolute the different ion profiles in the absence of the quadrupole - such as with a Faraday collector - the ions should ideally show a lot less curvature close to their respective cutoff points.

\section{Sinusoidal versus Square Waveform Operation}

For a given peak-to-peak amplitude, a square wave will have a larger $V_{\text {rms }}$ value than a sine wave by a factor of $\sqrt{2}$, so a digital square wave would be expected to offer more effective filtering than an equivalent $\mathrm{V}_{p-p}$ amplitude sine wave. The expected result is that at a given $\mathrm{rf}_{p-p}$ amplitude, a greater proportion of ions will be filtered with a square wave relative to a sine wave of the same amplitude (p-p). Sine and square

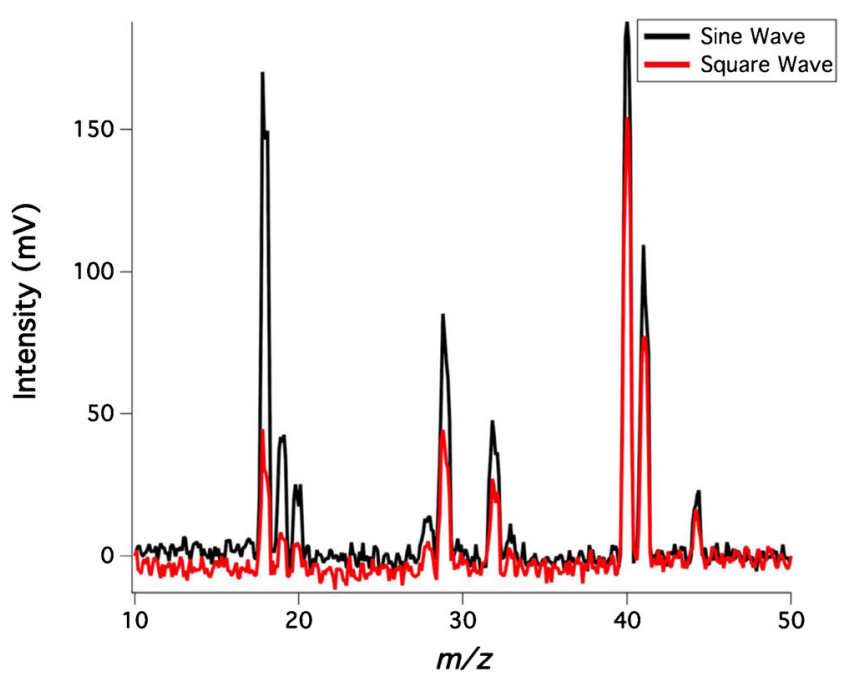

Figure 5. Comparison of ions transmitting through the LoebEiber mass filter operated with either a sinusoidal or square waveform. The linear quadrupole was scanned from $\mathrm{m} / \mathrm{z} 10$ 50 while the Loeb-Eiber mass filter was operated at a continuous frequency and amplitude of $50 \mathrm{MHz}$ and $2.5 \mathrm{~V} p-p$, respectively 
waves would not be expected to differ if operated at the same $\mathrm{V}_{\text {rms }}$ value. To evaluate the differences between sinusoidal and square waves, a $50 \mathrm{MHz}, 2.5 \mathrm{~V}_{p-p}$ signal was applied to the Loeb-Eiber filter (corresponding to fixed rf and LMCO conditions) while the linear quadrupole was scanned from $\mathrm{m} / \mathrm{z} 10$ 50. The glow discharge source was operated at $800 \mathrm{mTorr}$ Ar in current-limited-mode at $0.65 \mathrm{~mA}$. Figure 5 shows the differences in the transmitted ion signal through the Loeb-Eiber filter using the two different waveforms.

Compared with the transmitted ions observed using a sinusoidal waveform, the square waveform attenuates all the signals more effectively, as expected. It is also seen that there is an $\mathrm{m} / \mathrm{z}$ dependence on the degree of attenuation, with the smaller $\mathrm{m} / \mathrm{z}$ signals being significantly more attenuated than the larger $\mathrm{m} / \mathrm{z}$ values. This result indicates that the linear dependence on ion transmission holds for square waveforms as it does for sinusoidal waveforms. Thus, digital waveforms are a realistic possibility for future development.

\section{Conclusions}

By placing a Loeb-Eiber mass filter in front of a traditional linear quadrupole, we have been able to verify the operation of the Loeb-Eiber device as a high-pass mass filter, effectively verifying the operational principles laid out more than half a century ago. The new hybrid instrument has enabled us to verify several important claims regarding the operation of the Loeb-Eiber mass filter: (1) that ions can be effectively filtered at $\sim 1$ Torr, (2) that for ions of a fixed mass-to-charge ratio, the ion transmission current decreases linearly with increasing $\mathrm{rf}$ amplitude on the Loeb-Eiber mass filter, (3) that the cutoff voltage at which all ions of a particular $m / z$ value are effectively blocked is linearly related to mass-to-charge, and (4) that square waveforms can filter ions more effectively than sinusoidal waveforms for a given peak-peak rf amplitude.

\section{Acknowledgments}

Derrell L. Hood and Christine M. Fisher are acknowledged for their contributions in the early stages of this work at Ohio University. Dr. Guido F. Verbeck at University of North Texas assisted with the design of the chip-based mass filter. This work was supported by an NSF CAREER Award from the National Science Foundation (NSF) through grant numbers CHE 0745590 and CHE 1339153. Any opinions, findings, and conclusions or recommendations expressed in this article are those of the participants and do not necessarily reflect the views of NSF.

\section{References}

1. Badman, E.R., Cooks, R.G.: Miniature mass analyzers. J Mass Spectrom 35, 659-671 (2000)

2. Henry, C.M.: The incredible shrinking mass spectrometers. Anal Chem 71, 264A-268A (1999)

3. Ouyang, Z., Cooks, R.G.: Miniature mass spectrometers. Annu Rev Anal Chem 2, 187-214 (2009)
4. Smith, P.A., Jackson-Lepage, C.R., Koch, D., Wyatt, H.D.M., Hook, G.L., Betsinger, G., Erickson, R.P., Eckenrode, B.A.: Detection of gas-phase chemical warfare agents using field-portable gas chromaography-mass spectrometry systems: instrument and sampling strategy considerations. Trends Anal Chem 23, 296-306 (2004)

5. Sloan, K.M., Mustacich, R.V., Eckenrode, B.A.: Development and evaluation of a low thermal mass gas chromatograph for rapid forensic Gc-MS analyses. Field Anal Chem Technol 5, 288-301 (2001)

6. Eckenrode, B.A.: Environmental and Forensic applications of fieldportable GC-MS: an overview. J Am Soc Mass Spectrom 12, 683-693 (2001)

7. Grant, P., Chambers, D., Grace, L.: Phinney, D., Hutcheon, I.: Advanced techniques in physical forensic science. Physics Today 51, 33-38 (1998)

8. Transportation, Committee on Assessment of Security Technologies for Transportation: Opportunities to improve airport passenger screening with mass spectrometry. National Research Council, Washington (2004)

9. Science on Location: Forensic Science on the Move, Available at: Http:// www.Chem.Purdue.Edu/Docs/Forensicworkshopfinalreport.Pdf. Accessed September 1 (2014)

10. Cornish, T.J., Cotter, R.J.: High-order kinetic energy focusing in an end cap reflectron time-of-flight mass spectrometer. Anal Chem 69, 4615-4618 (1997)

11. Cotter, R.J., Fancher, C., Cornish, T.J.: Miniaturized time-of-flight mass spectrometer for peptide and oligonucleotide analysis. J Mass Spectrom 34, 1368-1372 (1999)

12. Wapelhorst, E., Hauschild, J.-P., Muller, J.: Complex MEMS: a fully integrated TOF micro mass spectrometer. Sens Actuators A 138, 22-27 (2007)

13. Fox, J., Saini, R., Tsui, K., Verbeck, G.: Microelectromechanical system assembled ion optics: an advance to miniaturization and assembly of electron and ion optics. Rev. Sci. Instrum. 80, 093302 (2009)

14. Taylor, S., Tunstall, J.J., Leck, J.H., Tindall, R.F., Jullien, J.P., Batey, J., Syms, R.R.A., Tate, T., Ahmad, M.M.: Performance improvements for a miniature quadrupole with a micromachined mass filter. Vacuum 53, 203206 (1999)

15. Taylor, S., Tindall, R.F., Syms, R.R.A.: Silicon-based quadrupole mass spectrometry using microelectromechanical systems. J Vac Sci Technol B 19, 557-562 (2001)

16. Turner, P.G., Dugdale, A., Young, I.S., Taylor, S.: Portable mass spectrometry for measurement of anaesthetic agents and methane in respiratory gases. Veterinary J 177, 36-44 (2008)

17. Hogan, T.J., Taylor, S., Cheung, K., Velasquez, L.F.: Performance characteristics of a mems quadrupole mass filter with square electrodes: experimental and simulated results. IEEE Trans Instrum Meas 59, 2458-2467 (2010)

18. Cheung, K., Velasquez-Garcia, L.F., Akinwande, A.: Chip-scale quadrupole mass filters for portable mass spectrometry. J Microelectromech Syst 19, 469-483 (2010)

19. Diaz, J.A., Giese, C.F., Gentry, W.R.: Sub-miniature ExB sector field mass spectrometer. J Am Soc Mass Spectrom 12, 619-632 (2001)

20. Louris, J.N., Cooks, R.G., Syka, J.E.P., Kelley, P.E., Stafford, G.C., Todd, J.F.J.: Instrumentation, applications, and energy deposition in quadrupole ion-trap tandem mass-spectrometry. Anal Chem 59, 1677-1685 (1987)

21. Blain, M.G., Riter, L.S., Cruz, D., Austin, D.E., Wu, G.X., Plass, W.R., Cooks, R.G.: Towards the hand-held mass spectrometer: design considerations, simulation, and fabrication of micrometer-scaled cylindrical ion traps. Int J Mass Spectrom 236, 91-104 (2004)

22. Pau, S., Pai, C.S., Low, Y.L., Moxom, J., Reilly, P.T.A.: Microfabricated quadrupole ion trap for mass spectrometer applications. Phys. Rev. Lett. 96, 120801 (2006)

23. Chaudhary, A., van Amerom, F.H.W., Short, R.T., Bhansali, S.: Fabrication and testing of a miniature cylindrical ion trap mass spectrometer constructed from low temperature co-fired ceramics. Int J Mass Spectrom 251, 32-39 (2006)

24. Badman, E.R., Johnson, R.C., Plass, W.R., Cooks, R.G.: A miniature cylindrical quadrupole ion trap: simulation and experiment. Anal Chem 70, 4896-4901 (1998)

25. Kornienko, O., Reilly, P.T.A., Whitten, W.B., Ramsey, J.M.: Micro ion trap mass spectrometry. Rapid Commun Mass Spectrom 13, 50-53 (1999)

26. Jesseph, A.V., Fox, J.D., Verbeck, G.F.: Ion isolation and collision-induced dissociation in a $0.5 \mathrm{~mm} \mathrm{R}(\mathrm{O})$ cylindrical ion trap. Int. J. Mass Spectrom 295, 149-152 (2010) 
27. Ouyang, Z., Badman, E.R., Cooks, R.G.: Characterization of a serial array of miniature cylindrical ion trap mass analyzers. Rapid Commun Mass Spectrom 13, 2444-2449 (1999)

28. Van Amerom, F.H.W., Chaudhary, A., Cardenas, M., Bumgarner, J., Short, R.T.: Microfabrication of cylindrical ion trap mass spectrometer arrays for handheld chemical analyzers. Chem Eng Commun 195, 98-114 (2008)

29. Cruz, D., Chang, J.P., Fico, M., Guymon, A.J., Austin, D.E., Blain, M.G.: Design, microfabrication, and analysis of micrometer-sized cylindrical ion trap arrays. Rev. Sci. Instrum. 78, 015107 (2007)

30. Lammert, S.A., Plass, W.R., Thompson, C.V., Wise, M.B.: Design, optimization and initial performance of a toroidal $\mathrm{rf}$ ion trap mass spectrometer. Int J Mass Spectrom 212, 25-40 (2001)

31. Lammert, S.A., Rockwood, A.A., Wang, M., Lee, M.L., Lee, E.D., Tolley, S.E., Oliphant, J.R., Jones, J.L., Waite, R.W.: Miniature toroidal radio frequency ion trap mass analyzer. J Am Soc Mass Spectrom 17, 916-922 (2006)

32. Austin, D.E., Wang, M., Tolley, S.E., Maas, J.D., Hawkins, A.R., Rockwood, A.L., Tolley, H.D., Lee, E.D., Lee, M.L.: Halo ion trap mass spectrometer. Anal Chem 79, 2927-2932 (2007)

33. Peng, Y., Hansen, B.J., Quist, H., Zhang, Z.P., Wang, M., Hawkins, A.R., Austin, D.E.: Coaxial ion trap mass spectrometer: concentric toroidal and quadrupolar trapping regions. Anal Chem 83, 5578-5584 (2011)

34. Taylor, N., Austin, D.: A simplified toroidal ion trap mass analyzer. Int J Mass Spectrom 321, 25-32 (2012)

35. Loeb, L.B.: The energy of formation of negative ions in $\mathrm{O}_{2}$. Phys $\mathrm{Rev} 48$, 684-689 (1935)

36. Eiber, H.: Determination of ion masses at gas pressures up to several millimeters by a screen grid charged with high frequency. Z Angew Physik 15, 461-463 (1963)

37. Hood, D.L.: Development of a novel Loeb-Eiber mass filter, development of a novel Loeb-Eiber mass filter. Ohio University (2009)
38. Hoffmann, W.D., Jin, F., Jackson, G.P.: A Loeb-Eiber mass filter for miniature mass spectrometry. In: Proceedings of the 62nd ASMS Conference on Mass Spectrometry and Allied Topics: Baltimore, MD (2014)

39. Bradbury, N.E., Nielsen, R.A.: Absolute values of the electron mobility in hydrogen. Phys Rev 49, 38-393 (1936)

40. Kimmel, J.R., Engelke, F., Zare, R.N.: Novel method for the production of finely spaced Bradbury-Nielsen gates. Rev Sci Instrum 72, 4354-4357 (2001)

41. Yoon, O.K., Zuleta, I.A., Robbins, M.D., Barbula, G.K., Zare, R.N.: Simple template-based method to produce Bradbury-Nielsen gates. J Am Soc Mass Spectrom 18, 1901-1908 (2007)

42. Zuleta, I.A., Barbula, G.K., Robbins, M.D., Yoon, O.K., Zare, R.N.: Micromachined Bradbury-Nielsen gates. Anal Chem 79, 9160-9165 (2007)

43. Sujatha, L., Goutham, M.S., Saravanan, M., Selvakumar, V.S.: Performance of silicon-on-insulator multiuser MEMS processes-based electrothermally actuated silicon microgrippers. J. Micro-Nanolithogr, MEMS MOEMS 12, 8 (2013)

44. Liu, J., Oakley, C., Shandas, R.: Capacitive micromachined ultrasonic transducers using commercial multi-user MUMPS process: capability and limitations. Ultrasonics 49, 765-773 (2009)

45. Khan, F., Bazaz, S.A., Sohail, M.: Design, implementation, and testing of electrostatic SOI-MUMPS based microgripper. Microsyst Technol 16, $1957-1965$ (2010)

46. Cowen, A., Hames, G., DeMaul, M., Wilcenski, S., Hardy, B.: SOIMUMPS Design Handbook; MEMSCAP Inc.: (2011)

47. Eiber, H.: Idenfifizierung Von Ionen in Verdunnten Gasen Mit Hilfe Eines Neuartigen Hochfrequenz Massensiebes, Compt. Rend. Conf. Intern. Phénomènes D’ionization Gaz, Paris pp. 305-308 (1963) 